\title{
TRUTH AND REPRESENTATION IN SCIENCE: TWO INSPIRATIONS FROM ART
}

\author{
Beyond Mimesis and Nominalism: Representation in Art and Science \\ London School of Economics and the Courtauld Institute, London 2006
}

\author{
Anjan Chakravartty \\ Institute for the History and Philosophy of Science and Technology, and Department of Philosophy \\ University of Toronto
}

\begin{abstract}
Realists regarding scientific knowledge - those who think that our best scientific representations truly describe both observable and unobservable aspects of the natural world - have special need of a notion of approximate truth. Since theories and models are rarely considered true simpliciter, the realist requires some means of making sense of the claim that they may be false and yet close to the truth, and increasingly so over time. In this paper, I suggest that traditional approaches to approximate truth are insensitive to two crucial features of scientific knowledge, and that for each of these, analogies between representational practices in the sciences and in art prove useful to understanding how this situation can be remedied. First, I outline two distinct ways in which representations deviate from the truth, commonly referred to as ‘abstraction' and 'idealization'. Second, I argue that these practices exemplify different conventions of representation, and that for each, the conditions of approximation relevant to explicating the concept of approximate truth must be understood differently. The concept is thus heterogeneous; approximate truth is a virtue that is multiply realized, relative to different contexts of representation. This understanding is facilitated, I suggest, by considering the distinction between realistic and non-realistic representation in art.
\end{abstract}

\section{Varieties of truth in art and science}

Not so long ago, pursuing the notion that the philosophies of art and science can inform one another in mutually productive ways might have been considered a cultured but rather fringe activity. Recently, however, philosophers more generally have awoken to the import of provocative and substantive analogies between practices of representation in these fields, and it is the spirit of this pursuit that motivates this essay. My primary concern here is to understand the nature of truth in the scientific context, and it will be my contention that this understanding, far from being a simple matter of mastering the T-schema, requires an appreciation of the distinction between two different conventions of representation - one associated with practices of abstraction, and the other with practices of idealization. It is here, I believe, that analogies to practices of representation in art can serve as valuable heuristics towards understanding how and in what manner scientific representations can be true. 
The term 'scientific representation' is commonly applied to many things, and would benefit from a more precise consideration than I can give it here. For present purposes, let me simply take such representations to include the usual items traditionally held to have representational status in the sciences, $v i z$. theories and models, however these things are best defined, and constituting the ontological categories commonly associated with them: linguistic and mathematical entities, computer simulations, concrete objects, and so on. Other key concepts here will of course include those of abstraction and idealization, and I will have something to say about each in turn. Let me begin, however, with the central concept whose explication this essay is intended to serve. Clearly, not all philosophers of science believe that the sciences are in the truth business, but an impressive diversity do, including different kinds of realists and empiricists. The former take the truths of science to include facts about unobservable entities and processes, and some of the latter acknowledge only truths about the observable, but all believe that scientific knowledge involves or at the very least aspires to substantive truths about the world, in some form or other. This is the first of two assumptions I will make here, at the outset.

The second is that descriptions of entities and processes afforded by scientific representations are generally false, strictly speaking. I will not argue for this here, but neither do I take it to be controversial. Even realists and empiricists who think that the sciences are in the truth business will readily admit the hyperbole involved in suggesting that current representations (however circumscribed) are generally, perfectly and comprehensively true. The history of the sciences has made a mockery of that suggestion in the past, and no doubt there is further mockery to come. It seems that anyone who endorses the idea of scientific truth as a reasonable aspiration requires some means of making sense of the idea that inaccurate representations can be close to the truth, and perhaps even get better with respect to truth over time. In the literature this requirement has motivated several accounts of "approximate truth", in terms of which, it is argued, one may understand such improvements. There would seem to be a widely held intuitive platitude concerning the notion of approximate truth, and Stathis Psillos (1999, p. 277) summarizes it well: 'A description $D \ldots$ is approximately true of [a state] $S$ if there is another state $S^{\prime}$ such that $S$ and $S^{\prime}$ are linked by specific conditions of approximation, and $D \ldots$ is true of $S^{\prime}$.' As it stands, however, the 
helpfulness of this statement is greatly impaired by the vagueness of the phrase 'conditions of approximation'. In essence, the remainder of this essay is an attempt to clarify this phrase.

I believe that the clarification required is wonderfully illuminated by drawing analogies to certain practices of representation in art, and as a final foreshadowing remark, let me mention briefly the pathbreaking work in this area that informs several of the thoughts to follow. Nelson Goodman (1976) is celebrated for presenting a detailed analysis of the "symbol systems" in terms of which different forms of art express their content. At the end of his book on the subject, Goodman (p. 262) says something particularly striking about the comparison between representations in art and in science:

...have I overlooked the sharpest contrast: that in science, unlike art, the ultimate test is truth? Do not the two domains differ most drastically in that truth means all for the one, nothing for the other? ... Despite rife doctrine, truth by itself matters very little in science.

It should be stated immediately that Goodman does not of course think that truth is unimportant in the sciences. Important truths about the natural world are indeed of great interest to scientists, and while one may admit that scientific laws are seldom true as they stand, we have an interest in 'arriving at the nearest approximation to truth that is compatible with our other interests' (1976, p. 263). Ultimately, says Goodman, truth can be understood in terms of 'a matter of fit' between theories and facts, and as it turns out, just this sort of "fitting" is characteristic of the relationship between art and the world. Truth in both domains should be understood in terms of approximating reality by means of representations.

The reason Goodman suggests that truth by itself matters little, is that truth amounts to nothing unless one, in addition to having truthful representations, is properly acculturated with the conventions of representation in terms of which they express their content. It is precisely these conventions that I take to constitute the 'conditions of approximation' whose explication is required in order to make sense of the notion of approximate truth, and so by exploring the former, I aim to shed light on the latter. In the 
following I will suggest that understanding two central features of scientific knowledge are crucial to illuminating these conditions of approximation, and it is here that analogies to representation in art may prove useful. The first of these features is the distinction between abstraction and idealization in connection with scientific representation, and the second concerns the nature and pragmatics of scientific practice. Let us consider these features in turn.

\section{Preliminaries on approximate truth}

A moment ago I suggested that generally speaking, knowledge contained within scientific representations is usually understood as approximately true at best. Three main families of accounts of approximate truth have emerged in the literature since the 1960s, and before considering the nature and relevance of abstraction and idealization in this context, it will serve us to have a synoptic overview of each of these approaches. I will refer to them respectively as the verisimilitude approach, due to Karl Popper, the possible worlds approach, formulated in different ways by authors including Pavel Tichý, Ilkka Niiniluoto, and Graham Oddie, and the type hierarchy approach, offered by Jerrold Aronson, Rom Harré, and Eileen Cornell Way.

Popper was the first to give a definition of what he called 'verisimilitude' or 'truth-likeness'. On his (1972, pp. 231-236) view, scientific theories within a domain may exhibit increasing levels of verisimilitude over time, and this relative ordering can be expressed as follows. Consider a temporallyordered sequence of theories concerning the same subject matter: $T_{1}, T_{2}, T_{3}, \ldots$ Now, for each of these theories, consider the set of all of its true consequences (for example, $T_{1}^{\mathrm{T}}$ ) and the set of all of its false consequences $\left(T_{1}^{\mathrm{F}}\right)$. A comparative ranking of the verisimilitude of any two theories can be given, suggests Popper, by comparing their true and false consequences. For any theory $T_{\mathrm{n}}$, and any previous theory $T_{<\mathrm{n}}, T_{\mathrm{n}}$ has a higher degree of verisimilitude than $T_{<\mathrm{n}}$ if and only if either of the following statements is true (' $\subseteq$ ' here stands for set-theoretic inclusion, and ' $\subset$ ' for proper inclusion): 
$1 \quad T_{<\mathrm{n}}^{\mathrm{T}} \subset T_{\mathrm{n}}^{\mathrm{T}} \quad$ and $\quad T_{\mathrm{n}}^{\mathrm{F}} \subseteq T_{<\mathrm{n}}^{\mathrm{F}}$

$2 \quad T_{<\mathrm{n}}^{\mathrm{T}} \subseteq T_{\mathrm{n}}^{\mathrm{T}} \quad$ and $\quad T_{\mathrm{n}}^{\mathrm{F}} \subset T_{<\mathrm{n}}^{\mathrm{F}}$

Though intuitive, this account is unfortunately afflicted by fatal difficulties, first described by David Miller (1974) and Tichý (1974). As it turns out, one can prove that in neither the case of 1 or 2 above can both conjuncts be satisfied together. On Popper's definition, in order that $T_{\mathrm{n}}$ have greater approximate truth than $T_{<\mathrm{n}}, T_{\mathrm{n}}$ would have to be true simpliciter. Thus, one false theory cannot have more approximate truth than another, and this rather defeats the aim of providing an understanding of what it could mean to rank false theories with respect to truth.

The precise formulation of the possible worlds approach (also called the 'similarity' approach) varies between different authors, but let me give a general characterization of it. The basic idea is first to identify the truth conditions of a theory with the set of possible worlds in which it is true, and then calculate what one may call 'truth-likeness’ by means of a function that measures the average “distance” between the actual world and the worlds in that set. In this way, one may generate an ordering of theories with respect to truth-likeness. For example, consider the class of atomic propositions entailed by a theory, each attributing a specific state to a particular; possible worlds here are described by distributions of truth values across these atomic propositions. The greater the agreement between a given theory and a theory correctly describing the actual world, the greater the former's truth-likeness. ${ }^{1}$ Though less clearly fatal than the objections to Popper's account, the possible worlds approach is itself subject to two important controversies. First, Miller (1976) argues that on this view, measures and relative orderings of truth-likeness are language-dependent: logically equivalent theories may have different degrees of truthlikeness depending on the language in which they are expressed, and the relative truth-likeness of two theories may be reversed when translated into another language with logically equivalent predicates. Second, Aronson (1990) shows that on this view, the truth-likeness of a proposition (whether true or

\footnotetext{
${ }^{1}$ See Tichý 1974, 1976, 1978, Niiniluoto 1984, 1987, 1999, and Oddie 1986a, 1986b, 1990. Niiniluoto 1998 summarizes the different formulations associated with this approach.
} 
false) depends on the number of atomic states under consideration, and it is at least questionable whether the truth-likeness of propositions concerning states of affairs other than that described by the proposition at issue should be relevant in this way.

Finally, a third approach to approximate truth analyzes truth-likeness in terms of similarity relationships between nodes in type hierarchies: tree-structured graphs of types and subtypes. ${ }^{2}$ The nodes represent concepts or things in the world, and links between them represent relations between concepts or things. As an illustration, consider the standard biological taxonomy of organisms divided into kingdoms, phyla, and so on down to species. Similarity here is defined with respect to locations within type hierarchies. In order to show that dolphins are more similar to whales than tuna, for example, one calculates their degrees of similarity to one another by means of a weighted difference measure, comparing the properties these types share and those in which they differ. Now consider an analogous comparison between a node in a theoretical type hierarchy and a corresponding node in the actual type hierarchy of the world. Truth-likeness is measured by the "distance” between a theoretical claim about a type and the correct description of that type, reflecting the degree of similarity of the nodes with which these descriptions are associated. The most striking difficulty with this approach is that it appears to require the existence of a unique type hierarchy of the world. Lacking this, it seems there is no determinate answer to the question of what a node in a theoretical type hierarchy should be compared to. As Psillos (1999, p. 277) observes, on this view, significantly different type hierarchies would lead to different measures of approximate truth, and the assumption that nature admits of only one correct taxonomy is controversial at best.

Each of the three approaches to approximate truth just outlined face interesting challenges, and it is not my intention here to see whether these specific challenges can be met. My goal in reviewing them is rather to set the stage for what I take to be a more general complaint, to be levelled against all three. None of these established approaches to approximate truth is particularly interested in the qualitative dimensions of the concept, which concern the ways in which theories and models typically diverge from

\footnotetext{
${ }^{2}$ See Aronson 1990, and Aronson, Harré, \& Way 1994, pp. 15-49.
} 
truth in the first place. It is precisely a deeper understanding of these details, I will contend, that is crucial to understanding the nature and truth content of scientific representation. And it is in this context that I will take inspiration from certain analogies to practices of representation in art.

\section{Truth in the context of abstraction and idealization}

Let us turn now to consider the ways in which scientific theories and models are constructed so as to deviate from the truth. There are, I believe, two such ways, and I will call them abstraction and idealization respectively. ${ }^{3}$ Roughly put, an abstract representation is the result of a process of abstraction; that is, one in which only some of the potentially many factors that are relevant to the behaviour of a target system are built into the representation. In such a process other parameters are ignored, either intentionally or unwittingly, so as to permit the construction of a tractable representation. A commonly discussed example of this is the model of the simple pendulum. Here, among other simplifying assumptions made in the construction of the model, one simply omits the factor of frictional resistance due to air.

On the other hand, an idealized representation is the result of a process of idealization; that is, one in which at least one of the parameters of the target system is represented in a way that constitutes a distortion or a simplification of its true nature. In such a process, one is not excluding parameters, as in abstraction, but incorporating them, again either intentionally or unwittingly, in such a manner as to represent them in ways they are not - indeed, perhaps in ways they could not possibly be. Idealized representations thus furnish strictly false descriptions of their counterparts in the world. For example, in the Principia, Newton assumes that the sun is at rest in his derivation of Kepler's laws of planetary motion. According to his own theory, however, this would require that the sun have infinite mass, and Newton clearly did not believe this to be the case. On his understanding, the sun experiences small

\footnotetext{
${ }^{3}$ Recent reflections on this distinction are numerous. For early and more recent examples of the most comprehensive discussions, see McMullin 1985 and Jones 2005.
} 
amounts of motion as a consequence of the attractions of other bodies, and so, the "attribution" of infinite mass constitutes an idealization.

Abstraction and idealization are not mutually exclusive processes, and consequently, representations are often both abstract and idealized. Now, given these two practices of deviation from the truth, how should one think about the approximate truth of scientific representations? It seems to me that there is a straightforward answer to this question in cases of pure abstraction - that is, cases of abstraction which incorporate no idealization - and a less obvious answer in cases of idealization. In the former cases, there seems to be no impediment to thinking of pure abstractions as true simpliciter, if only in connection with a certain class of target systems. Pure abstractions correctly describe certain features of things in the world, even if they do not describe all of the properties and relations potentially relevant to the phenomena at issue. It is natural, of course, to view abstractions as yielding false descriptions because of their omissions. But in the case of pure abstractions, where no idealization is involved, target systems in which only those parameters represented contribute to the behaviour of the system are presumably possible; if they were not, this would indicate the presence of an idealization. Therefore, pure abstractions are perfectly accurate representations of some nomically possible target systems, even if they are impoverished representations of other, more complex ones. Clearly, one may apply a pure abstraction to a more complex system it does not correctly describe, but this should not be taken to discredit the truth of the representation. Neglecting air resistance usually counts as an error of omission, but it would not be in connection with a system in a vacuum.

This suggests a straightforward articulation of the notion of approximate truth qua abstraction. Consider all of the parameters potentially relevant the behaviour of a particular target system. Degrees of approximate truth are correlated here with the extent to which representations incorporate these parameters. The greater the number of factors built into the representation, the greater its approximate truth. This suggestion for assessing relative degrees of approximate truth does justice, I think, to the intuition that higher degrees of abstraction correspond to lesser degrees of truth, but without failing to appreciate that abstractions may yet characterize some things perfectly accurately. Pure abstractions yield 
correct descriptions of a certain class of target systems while being more or less approximately true in application to others, and here we have our first insight into what 'conditions of approximation' means in the analysis of approximate truth. In these cases, conditions of approximation can be understood simply in terms of how much information a representation yields, or its comprehensiveness, relative to a specific kind of target system, or class of systems.

In cases of idealization, however, one requires a rather different understanding of the relevant conditions of approximation. For here, unlike in cases of pure abstraction, one does not have the luxury of representations that accurately characterize at least some, nomically possible phenomena. Idealizations are more egregiously fictional than abstractions; they constitute not mere omissions, but distortions of things in the world. Models in classical mechanics, for example, generally treat the masses of bodies as though they are concentrated at extensionless points, but given the nature of mass as we understand it, it cannot be concentrated this way in any world such as ours, where particulars with masses exist. What information about the world is contained in fictions such as these?

A failure to grapple seriously with the qualitative nature of idealization is, I believe, a defect of extant accounts of approximate truth. Consider my illustration earlier of the possible worlds approach, in which one considers the class of atomic propositions entailed by a theory, each attributing a specific state to a particular, as a means towards evaluating its approximate truth. In cases of pure abstraction, one may justifiably claim here that the greater the extent to which a representation yields true descriptions of systems in the world, the greater its truth-likeness. Idealizations, however, do not generally give true descriptions of atomic states of affairs, for they are constructed in such a way as to characterize their objects in a distorted manner. Likewise, consider again the type hierarchy approach, where one calculates degrees of similarity between theoretical propositions and true ones by performing weighted difference measures involving the properties these propositions describe in common and those in which they differ. Arguably, however, idealized characterizations may not describe any properties in common with true theories, because they correctly describe fictional properties, not actual ones. The conditions of 
approximation relevant to assessing approximate truth qua idealization must be understood differently, I think, than the relevant conditions of approximation qua abstraction.

\section{Denotation in art, reference in science}

In order to appreciate how idealizations bear on the notion of approximate truth, let me now return to Goodman, and draw a first analogy to representation in art. In section 1, I quoted Goodman as suggesting that in both art and the sciences, successful representation is a matter of fitting or approximating things in the world. Let us now consider this suggestion in more detail, beginning with an examination of Goodman's reflections on the nature of realistic and non-realistic representation. It is precisely this distinction, I will argue, that is important to understanding of the "truth content" of idealized theories and models. The contrast between the nature of this content in cases of abstraction and idealization will provide crucial insight into how different contexts of representation call for a flexible approach on the part of those seeking to explicate the concept of approximate truth.

In the opening sections of his major work on artistic representation, Goodman (1976, p. 34) considers the question of how best to understand the nature of realism in this context. His answer appears at first both provocative and negative: 'Surely not...any sort of resemblance to reality.' Goodman does not provide much help with the ambiguous term 'resemblance', here, but on any obvious reading, his answer presents a prima facie puzzle of interpretation. If one interprets 'resemblance' narrowly to mean 'similarity in appearance', this might seem a strange claim regarding much art, though not perhaps regarding science; in the latter case one hardly expects mathematical equations (for example) to share similarities in appearance with acids and bases and populations of organisms. Reading 'resemblance' more broadly, as 'having some feature or features in common', the puzzle of interpretation extends even to the scientific case, since most philosophers of science hold that at least some parts of our best theories and models do, in fact, have features in common with their target systems, such as commonalities in structure (whether observable or unobservable). These interpretive puzzles, however, may be resolved with the clarification that for Goodman, realism is by no means inconsistent with resemblance in either of 
the senses just mentioned. His point is rather to emphasize that, as suggested earlier, realism of representation is only achieved in special circumstances, viz. those in which agents considering the representation are aware of, or have been acculturated with, the system or systems of representation that have been employed in constructing it.

Consider a realistic picture, painted in ordinary perspective and normal colour, and a second picture just like the first except that the perspective is reversed and each colour is replaced by its complementary. The second picture, appropriately interpreted, yields exactly the same information as the first. And any number of other drastic but information preserving transformations are possible. Obviously, realistic and unrealistic pictures may be equally informative; informational yield is no test of realism.... The two pictures just described are equally correct, equally faithful to what they represent, provide the same and hence equally true information; yet they are not equally realistic or literal. ... Just here, I think, lies the touchstone of realism: not in quantity of information but in how easily it issues. And this depends upon how stereotyped the mode of representation is, upon how commonplace the labels and their uses have become. (Goodman 1976, pp. 35-36)

Goodman is a conventionalist about systems of representation: anything can represent anything else, subject to appropriately internalized conventions. As a consequence, one and the same representation can be realistic or not, depending on whether the relevant conventions have been internalized by the viewer or user.

Several fascinating issues concerning conventionalism and representation are raised by this and surrounding passages, but for the sake of present purposes, let me simply extract one key point: an understanding of the relevant and potentially different conventions of reading information from representations is crucial to how one understands that information. This point bears directly on my 
contention that if one is to have a genuinely informative account of what it means to say that scientific representations are approximately true, one must understand the different conditions of approximation exemplified by abstraction and idealization. These different conditions, I will suggest, should be understood in terms of different conventions of representation.

One last point arising from Goodman's discussion of artistic representation will prove useful in heading towards this conclusion. Goodman (1976, p. 5) suggests that 'the core of representation' is denotation. That is, in order for $x$ to represent $y, x$ must be a symbol for, or stand for, or refer to $y$. Symbols here include 'letters, words, texts, pictures, diagrams, maps, models, and more’ (1976, p. xi). In Goodman's terms, denotation is a species of reference, pointing from representations to things represented. And with this in mind, here finally is the first feature of artistic representation that I believe furnishes a provocative analogy for those hoping to understand the nature of approximate truth in science: just as in the case of art, where successful representation can be a function of denotation, in the sciences, successful representation can be a function of reference, even when theories and models offer idealized descriptions of the properties and relations of their target systems. Let us consider this suggestion in some detail.

To be sure, emphasizing reference is hardly novel in the philosophy of science, especially in discussions of scientific realism. Entity realists are especially well known for this, holding that under conditions in which one has significant evidence of an ability to manipulate or otherwise systematically exploit the causal properties of entities, one has good reason to believe that such entities exist, even while withholding belief from the theories that describe them. It is for this reason that entity realism can be cast as a response to challenges to realism posed by the history of science, which provides ample evidence of theoretical descriptions changing over time. Hacking (1983, chapter six), for example, contends that one may continue to refer to the same causal entity despite changes in the theories that describe it, and this furnishes a stable point around which realists can organize their knowledge claims regarding unobservables. Despite the fact that theories are false and likely to change, says the entity realist, there are conditions under which one has good reason to think that unobservable terms refer, and will continue 
to refer. Interestingly, the importance of relations of reference has never really shaped thinking about approximate truth, but it is my contention here that they are clearly relevant to understanding the differential truth content of pure abstractions and idealizations. Insofar as true (that is, non-idealized) claims about entities and processes can be extracted from idealizations, these are for the most part claims of successful reference, not the more detailed descriptions of target systems that one may associate with cases of pure abstraction.

This should not be taken to suggest, of course, that merely appealing to reference can save realist blushes. This appeal on behalf of entity realism is controversial, even amongst scientific realists. Many question whether it is coherent to be a realist merely about certain entities described by theories, since causal manipulations and exploitations seem to be based on further and substantial parts of those theories. And as I have argued elsewhere (2007, chapter 2), there does seem to be something anachronistic in the suggestion that scientists from different periods in the history of scientific investigation into an entity all believe in the same thing. In order to be more compelling, the realist's story must be told at a deeper level, with respect to specific properties and relations on which existential claims are based, and that are likely to survive (if only as limiting cases) in theories over time. Despite what I take to be serious difficulties, however, there is an important insight at the heart of entity realism nevertheless: degrees of belief in unobservable entities are generally and rightly correlated with the extent of one's causal contact with those entities. There are no stronger grounds for belief in an entity than an impressive ability to systematically exploit its causal properties, and less impressive abilities rightly ground more attenuated belief. On the impressive side of this continuum, claims of reference are concomitantly strong. For those of a strict empiricist bent, the same point can be made, mutatis mutandis, regarding observable entities.

Having emphasized the notion of reference, and having at least gestured towards some of nuances that must be taken into consideration concerning reference in this context, I am now in a position to describe what it means for one theory to be more or less approximately true than another qua idealization, and to contrast this with cases of pure abstraction. When it comes to truth, even the best idealizations contribute primarily existential claims. This does not mean, however, that all idealizations are on a par 
when it comes to the approximate truth of the more substantive descriptions they provide. Some idealizations approximate true descriptions of properties and relations better than others, and this is an important consideration in assessing their relative approximate truth. The relevant notion of approximation here is usually specified mathematically. One can define mathematically how Newtonian descriptions of certain properties approximate those of special relativity, for example, by showing how the equations of Newtonian mechanics are limiting cases of relativistic equations. The ideal gas law assumes that molecules of gas are point particles and that there are no forces of attraction between them, but it is possible to take into account both the space occupied by molecules of gas and small forces of mutual attraction. Thus, while the van der Waals equation generates values for various properties that approach those given by the ideal gas law at lower pressures (larger volumes), it yields different, more accurate values at higher pressures (smaller volumes). ${ }^{4}$ The Van der Waals equation, over certain ranges of pressure, volume, and temperature, describes the natures of these properties and their relations more accurately than the ideal gas law.

Earlier I credited Psillos with a precise statement of what I take to be a widely-held intuitive platitude regarding approximate truth, to the effect that a description is approximately true of a state if it can be 'linked by specific conditions of approximation' to a true description. It was precisely because of a lack of clarity regarding the question of what 'conditions of approximation' might mean that I undertook to focus attention there, with the goal of generating a more satisfying explication of the concept of approximate truth. I believe the various pieces of the puzzle are now to hand. When representations deviate from true characterizations of their target systems, they do so via abstraction, or idealization, or in many cases both. I have argued that insofar as representations are abstract, approximate truth may be gauged in terms of the numbers of potentially relevant features of their target systems they incorporate, so that theories incorporating greater numbers of these features may be thought of as more approximately true than those incorporating fewer. Pure abstractions yield descriptions of properties and relations that are true simpliciter of certain classes of target systems, and that may be more or less approximately true in

\footnotetext{
${ }^{4}$ McMullin 1985 contains a nice discussion of this (p. 259) and similar cases .
} 
application to others. The notion of approximate truth qua abstraction is thus simply the notion of comprehensiveness, generally assessed in connection with a specific target system, and the relevant condition of approximation here is the extent to which the numbers of factors incorporated into a representation match up with those in the target systems to which it is applied.

The notion of approximate truth qua idealization is importantly different, for here the issue is not the comprehensiveness of representations, but the accuracy with which they characterize the natures of the specific parameters they represent. Unlike pure abstractions, idealizations do not generally offer true characterizations of the properties and relations they concern, even if they do permit ontological claims, in virtue of successful reference. By reducing the number of idealized assumptions or the extent to which they idealize - by de-idealizing - one describes target systems in ways that admit of greater degrees of approximate truth. Unlike the case of abstraction, however, where improving a representation is simply a matter of increasing the number of potentially relevant factors it incorporates, there is no reason to expect that processes of de-idealization should follow any common pattern from one domain of theorizing to the next. There are many ways of incorporating idealized assumptions into representations, and the ways in which one describes possible de-idealizations may vary in just the way that idealizations do. In any case, whatever these variations, idealized representations may be improved in ways determinable in specific instances. Approximate truth qua idealization concerns the degree to which a representation resembles a non-idealized representation of the same target system, where degrees of resemblance are defined in specific cases. The relevant condition of approximation here is not comprehensiveness, but degrees of distortion or simplification of the specific properties and relations targeted.

Let me sum up the import of the first analogy furnished by representation in art before turning to a second. When viewing a painting or a sculpture, one may extract more or less information regarding the things it represents, depending on the extent of its realism. At one end of this spectrum is what Goodman calls realistic representation in art, or depiction. Here, the viewer is sufficiently acculturated with some relevant system or systems of representation to derive significant information about the subject matter represented. At the other end of the spectrum representations may convey very little information, but 
information nonetheless. Consider the representational content of paintings, for example. Just one of the reasons Pablo Picasso's Guernica (1937) is one of the most famous artworks of the twentieth century is its captivating representational power. Its subject is the bombing of the Basque town of Guernica by Hitler's and Mussolini’s air forces, with the complicity of Franco, during the Spanish Civil War. Aspects of the work, such as the figures of a bull, a dead baby in the arms of a screaming woman, a speared horse, the broken body of a soldier, and so on, represent various things with greater and lesser degrees of realism. The painting taken as a whole also has representational content. Among other things, for instance, it represents the rising threat of European fascism. ${ }^{5}$ Insofar as the painting represents this, however, it is not depictive, but merely denotative. It does not provide much in the way of “description” beyond the existential "claim” it makes concerning the presence of a terrifying danger.

Scientific representations also yield information about their subject matter, but whether they do so by providing true characterizations of specifically chosen parameters, or by distorting the parameters to which they successfully refer, will depend on how abstract and idealized they are. The contrast between depiction and mere denotation as a central feature of representation in art is an analogy for the contrast between truth and mere reference as a central feature of representation in the sciences. Higher degrees of approximate truth can be understood in terms of improved representations of the natures of target systems in the world, and this improvement can be mapped along two dimensions: how many of the relevant properties and relations one describes (abstraction), and how accurately one describes them (idealization). This simple formula, combined with an understanding of the conditions of approximation involved in the practices it describes, comprises an explication of the principal notions at stake in making sense of the idea of approximate truth.

\section{Representations and practice, as products and production}

Let me now turn, finally, to the second analogy between representation in art and science I promised at the start. This one also concerns approaches to truth, but in a rather different way than the

\footnotetext{
${ }^{5}$ See Suarez 2003, p. 236, for a discussion of this painting and associated literature.
} 
first. For quite apart from the question of whether one can make sense of the notion of approximate truth, it should be noted that in the philosophy of science, there is no consensus regarding what sorts of scientific claims one ought reasonably to regard as true or approximately true in the first place. While realists defend the reasonableness of believing scientific claims concerning both observable and unobservable aspects of target systems, various critics, including varieties of empiricists and instrumentalists, accept only the former. My goal in this final section is to employ a second analogy of representation between art and science so as to extend a bridge between these opposing camps. In part because of their obsession with unobservable things, realists are often guilty, I believe, of failing to note the significance of the observable. Certainly, realists like everyone else regard observable consequences as furnishing tests of the accuracy of representations, but I have something else in mind here. In scientific practice, one is often primarily concerned with whether and to what extent theories, models, procedures, tests, etc. work. Can we use them to make faster computer chips, manage eco-systems, and successfully complete the astounding variety of tasks associated with laboratories and fieldwork across the globe every day? Success in practice is measured in terms of observable consequences, and there is a strong current of pragmatism built into everyday scientific pursuits. The pragmatist's test of epistemic significance is utility, and utility is assessed by means of observables.

Antirealists often intimate that realist interpretations of scientific knowledge are out of touch with the everyday worlds of real science, as opposed to the rarefied philosophical worlds of imagined science. The prevalence of empirically adequate idealizations and pure abstractions applied to systems they do not correctly describe serves to fuel this scepticism. It is for this reason that the idea of approximate truth, and more specifically, the idea that different sorts of truths may be contained within different sorts of scientific representations, is so important to realism. Armed with an understanding of the truth content of both idealizations and pure abstractions applied to systems they do not correctly describe, one may connect desiderata that sceptics generally believe to be independent of one another: the generation of observable predictions within acceptable margins of error (the goal of much scientific endeavour); and the uncovering of facts regarding unobservables that underlie these predictions. In section 1, I suggested that 
a consideration of two important features of scientific knowledge would facilitate an account of the concept of approximate truth. The first of these was the distinction between abstraction and idealization. The second concerns the pragmatic dimensions of scientific practice, and this topic, I believe, is intimately connected to the first. Let me move on to the second, now, by means of a second analogy to representation in art.

The history of twentieth-century art is, to a great extent, the history of the avant-gardes and their forms of “abstraction”. Realistic conventions of representation, in Goodman's sense, were supplemented by varieties of experiments seeking to realize different sorts of conventions, both in the service of representation and even, in some cases, in the service of non-representational expression. These experiments initiated traditions that we now recognize as familiar artistic movements such as Cubism, Surrealism, Constructivism, and Abstract Expressionism. If there is anything like a unifying theme to be found in the disparate approaches of the avant-gardes, it is, perhaps, the increasing focus on processes of art production, as opposed to the visual properties of the products of these processes. Many of these artistic pioneers were self-consciously and primarily interested in reflecting on the nature of artistic representation itself, paying great attention, for example, to the nature of the canvass as a twodimensional surface, as opposed to the task of realistically representing three-dimensional subjects. This is one, partial interpretation of the motivations of analytic Cubism, but it is also a recurring theme in other movements. In the Russian tradition, Malevich’s Suprematism emphasized the materiality of the process of painting, as opposed anything like realistic representation. The surface of the canvass, its shape, the thickness of the paint, and so on, take on a new significance. Co-opting the slogan of the American art critic Clement Greenberg (2003/1939, p. 539), this is ‘art for art’s sake’.

The rise of performance art may exemplify this tendency towards attaching greater significance to processes involved in the creation of art as opposed to its products per se better than anything else. Some of my favourites include works associated with the Fluxus movement, such as Yoko Ono’s Cut Piece, performed twice, once in Tokyo (1964) and once in New York (1965). During these events, Ono sat on a stage while members of the audience approached, individually and in succession, to cut pieces of clothing 
from her body with a pair of scissors. Here as in all work in the performance art genre, the idea of a process takes on so much significance that it now is the central focus of the artwork. What matters is an event or a series of events. The idea that the value of the performance resides in any further output is completely lost. Of course, photographs of works of performance art are very important for purposes of discussion and art criticism, but such outputs are considered mere documents of the art form, not things that are important in their own right, and certainly not things that are the proper focus of attention when considering the nature or significance of the work.

Keeping in mind this analogy of a transition in focus from products to production, let us now return to the case of the sciences, and give due consideration to the pragmatic dimension of scientific practice. Focusing on processes of production led artists to a dizzying array of less-than-realistic representations. Analogously, focusing on processes of detection, experiment, and the innumerable tasks constituting everyday scientific work leads scientists to create ingenious abstractions and idealizations. In the successful pursuit of much of this work, one does not require anything like truth simpliciter. One of the main reasons abstractions and idealizations are so ubiquitous in the sciences is that they facilitate these tasks so well, within the degrees of accuracy and precision required in the contexts of particular scientific endeavours. Indeed, it is often the case that less approximately true representations are preferred to more approximately true ones. For while both may generate predictions that are adequate to specific endeavours, simpler though less approximately true theories and models are, generally, more easily taught, learned, and used.

Indeed, the epistemic virtues of inaccurate representations often extend beyond their mere contextual adequacy. One routinely applies pure abstractions and idealizations to phenomena whose properties and relations they do not correctly describe, but that is not to say that in such cases, representations yield no truths. ${ }^{6}$ The classical theory of gases idealizes several properties of gas molecules and their relations to one another, but nevertheless has the (putatively) true consequences that there are molecules composing gases, and that they have properties such as mass. These are truths about

\footnotetext{
${ }^{6}$ I owe thanks to Martin Thomson-Jones and Juha Saatsi for pressing this point with examples.
} 
particulars and properties that follow immediately from successful reference, but other truths stemming from idealization arguably go further. Frictionless surfaces are ideal, but models of spherical objects rolling down frictionless inclined planes correctly describe the motions of these objects as linear. Newtonian models of the earth-moon system are ideal, but correctly represent the mass of the earth as being greater than that of the moon. Idealizations generate substantially less truth simpliciter than pure abstractions, but what truths they do yield may nevertheless add to their pragmatic utility.

The emphasis on production as opposed to products in art and science has an echo in the intellectual traditions that study these practices. A delightful symmetry can be found, for example, in the juxtaposition of twentieth-century art criticism and post-positivist philosophy of science. One of the recurring themes of critiques of logical positivism was that it was too absorbed with normative projects based on rational reconstructions of the products of the sciences, such as theories and models, and as a consequence, it is argued, the positivists found themselves out of touch with actual scientific practice. Thus it comes as no surprise that the demise of positivism in the twentieth century was accompanied by the rise of the history of science as an essential tool for philosophers. Much post-positivist philosophy of science takes the details of scientific practice as its focus, thereby de-emphasizing considerations of the epistemic status of its products. And thus, the word ‘truth' does not appear in Kuhn's iconic essay in the history and philosophy of science, The Structure of Scientific Revolutions, and Hacking (1983) is ultimately more interested in intervening in the natural world than representing it.

This sort of pragmatism is something that realists must take to heart in grappling with the concept of approximate truth. It is a concept that is differently instantiated by means of different representational relationships, involving true descriptions of properties and relations in some cases, and little more than successful reference in others. Some representations are purely abstract, in which case they yield a multitude of true descriptions of certain classes of phenomena. Other representations are heavily idealized, and consequently their truth rests primarily in existential claims, and in the extent to which their descriptions of properties and relations measure up to true descriptions, in ways specifiable in connection with specific target systems. Most cases of scientific representation are neither pure abstractions nor pure 
idealizations, of course, but rather mixtures of both, in different proportions and to varying degrees. The concept of approximate truth is thus heterogeneous, to be explicated as may be appropriate in particular cases, within the myriad contexts of representation to which it may be applied.

It is thus the conclusion of this paper that in the sciences, approximate truth is best understood as a virtue that is multiply realized by means of different kinds of representational relationships between scientific products such as theories and models on the one hand, and target systems in the world on the other. $^{7}$ These different conventions of representation reflect the degrees to which theories and models abstract and idealize, and as a consequence, anyone hoping to understand the ways in which they approximate truth must take these conventions into serious consideration. In this and perhaps other ways, those interested in the nature of scientific knowledge have things to learn from the nature of representation in art.

\section{References}

Aronson, J. L. 1990: 'Verisimilitude and Type Hierarchies’, Philosophical Topics 18: 5-28.

Aronson, J. L., R. Harré, \& E. C. Way 1994: Realism Rescued: How Scientific Progress is Possible. London: Duckworth.

Chakravartty, A. 2007: A Metaphysics for Scientific Realism: Knowing the Unobservable. Cambridge: Cambridge University Press.

Goodman, N. 1976: Languages of Art: An Approach to a Theory of Symbols, $2^{\text {nd }}$ edition. Indianapolis: Hackett.

Greenberg, C. (2003/1939): 'Avant-Garde and Kitsch’, in C. Harrison \& P. Wood (eds.), Art in Theory, 1900-2000: An Anthology of Changing Ideas. Oxford: Blackwell.

Hacking, I. 1983: Representing and Intervening. Cambridge: Cambridge University Press.

\footnotetext{
${ }^{7}$ For a more leisurely route to this conclusion, see Chakravartty 2007, Part III.
} 
Jones, M. R. 2005: 'Idealization and Abstraction: A Framework', in M. R. Jones \& N. Cartwright (eds.), Idealization XII: Correcting the Model, Poznan Studies in the Philosophy of the Sciences and the Humanities 86: 173-217.

McMullin, E. 1985: ‘Galilean Idealization’, Studies in History and Philosophy of Science 16: 247-273.

Miller, D. 1974: 'Popper’s Qualitative Theory of Verisimilitude', British Journal for the Philosophy of Science 25: 166-177.

Miller 1976: 'Verisimilitude Redeflated', British Journal for the Philosophy of Science 27: 363-380.

Niiniluoto, I. 1984: Is Science Progressive? Dordrecht: D. Reidel.

Niiniluoto, I. 1987: Truthlikeness. Dordrecht: D. Reidel.

Niiniluoto, I. 1998: 'Verisimilitude: The Third Period', British Journal for the Philosophy of Science 49: $1-29$.

Niiniluoto, I. 1999: Critical Scientific Realism. Oxford: Clarendon.

Oddie, G. 1986a: 'The Poverty of the Popperian Program for Truthlikeness’, Philosophy of Science 53: 163-178.

Oddie, G. 1986b: Likeness to Truth. Dordrecht: D. Reidel.

Oddie, G. 1990: 'Verisimilitude by Power Relations', British Journal for the Philosophy of Science 41: 129-135.

Popper, K. R. 1972: Conjectures and Refutations: The Growth of Knowledge, $4^{\text {th }}$ edition. London: Routledge \& Kegan Paul.

Psillos, S. 1999: Scientific Realism: How Science Tracks Truth. London: Routledge.

Suárez, M. 2003: 'Scientific Representation: Against Similarity and Isomorphism’, International Studies in the Philosophy of Science 17: 225-244.

Tichý, P. 1974: ‘On Popper’s Definitions of Verisimilitude’, British Journal for the Philosophy of Science 25: 155-160.

Tichý, P. 1976: 'Verisimilitude Redefined', British Journal for the Philosophy of Science 27: 25-42.

Tichý, P. 1978: 'Verisimilitude Revisited', Synthese 38: 175-196. 